\title{
Upper and lower neural tube defects: an alternate hypothesis
}

\author{
Berdj H Garabedian, F Clarke Fraser
}

\begin{abstract}
It has been suggested that neural tube defects (NTDs) of the upper type (anencephaly, encephalocele, and thoracic spina bifida) may have a pathogenesis different from those of the lower type (lumbosacral spina bifida), since recurrent cases within a sibship were said always to be concordant with respect to NTD type. Also, spontaneous abortion, additional malformation, and recurrence rate were observed to be higher in the upper group, and there was an excess of females in upper NTD probands. To test this hypothesis, we measured the above variables in upper and lower NTDs in a sample from Quebec.

We found less than full concordance $(50 \%)$ of NTD type in 18 sib pairs. Recurrence rate was not significantly lower in the lower NTD group $(5.6$ v $5.8 \%)$. The other variables were in general agreement with previous studies, inconsistent findings possibly attributable to different NTD population incidences. These findings can be accounted for if upper and lower NTDs share a similar pathogenesis and the embryo is more susceptible during early than late neural tube formation. (f Med Genet 1993;30:849-51)
\end{abstract}

Incomplete closure of the neural tube early in vertebrate embryonic development results in a neural tube defect (NTD). Failure of closure in the head region results in anencephaly (AN) or encephalocele (EN); an NTD that occurs lower down is termed spina bifida cystica (SB). This purely anatomical distinction has been challenged by the hypothesis of Toriello and Higgins, ${ }^{1}$ based on a study from Michigan, that upper defects in humans are pathogenetically different from lower ones, the former arising from defective primary neurulation and the latter from abnormal canalisation (secondary neurulation).

Primary neurulation consists of induction of the neural plate and the raising and apposition of the neural folds, followed by their fusion. ${ }^{2}$ The final event in this phase of neural tube development is closure of the posterior neuropore. Secondary neurulation occurs caudal to the posterior neuropore and is an entirely different process. From the remnant of the primitive streak, mesenchymal cells emerge and condense. This mass of condensed cells is then canalised to form the caudal portion of the neural tube. ${ }^{2}$

The hypothesis was based on the observations that (1) in the families reported by Toriello and Higgins, ${ }^{1}$ all nine recurrent cases of
SB within the same sibship were concordant for type of SB (that is, upper or lower), and (2) mothers had more spontaneous abortions and probands had more additional malformations in the upper group. A study by Hall et $a^{3}$ on a sample from British Columbia (BC) showed, in addition, that NTD recurrence rate was higher in the upper group. Furthermore, Seller ${ }^{4}$ found an excess of females in upper NTD probands.

The point at which neurulation ends and canalisation begins in humans is not clear. On the basis of a study by Lemire et al, ${ }^{5}$ Toriello and Higgins ${ }^{1}$ assigned it to the level of the 11 th thoracic vertebral segment (T11); they designated SBs at T11 and above as upper and those at T12 and below as lower. Because of the known heterogeneity within the AN group, ${ }^{6}$ Toriello and Higgins ${ }^{1}$ excluded AN from their study. However, Hall et $a l^{3}$ included AN on the grounds that it arises from defective primary neurulation, whatever the cause.

To test the Toriello and Higgins hypothesis, the above variables were measured in a sample from the Quebec population.

\section{Methods}

Family histories of probands with a nonsyndromic NTD with or without other malformations were obtained from charts of NTD probands referred to the genetics unit of The Montreal Children's Hospital. NTDs at T11 and above were classified as upper NTDs and those involving only T12 or below as lower NTDs (following classification III of Hall et $\left.a l^{3}\right)$. When the level of the lesion was not indicated or not stated in sufficient detail in the genetics charts, patients' hospital charts were retrieved for inspection of radiographic reports.

Cases with additional malformations, but not conforming to a recognised syndrome, were included to make our sample comparable to those of Toriello and Higgins ${ }^{1}$ and of Hall $e t$ $a l^{3}$ and because those with additional malformations did not differ from those without them in the variables examined. Although Khoury et al suggested that NTDs with additional malformations were a distinct subclass on the basis of differences in epidemiological characteristics, no such distinction was seen in other studies. ${ }^{89}$

NTD recurrence rates in sibs of NTD probands were calculated using the single ascertainment method. ${ }^{10}$ Sex ratios in NTD probands were calculated as the number of males divided by the number of females. Spontaneous abortion rates were obtained by counting recognised fetal losses before 26 
Table 1 Recurrence rates in sibs of probands with upper or lower NTD.

\begin{tabular}{lccccccc}
\hline & \multicolumn{2}{c}{ Upper NTD } & & \multicolumn{2}{c}{ Lower NTD } & \\
\cline { 2 - 3 } & $\%$ & No & & $\%$ & No & & p \\
\hline Quebec & & & & & & \\
(present study) & 5.8 & 258 & & 5.6 & 249 & NS \\
BC $^{3}$ & 3.3 & 424 & & 0.7 & 277 & $<0.05$ \\
\hline
\end{tabular}

weeks of gestation and dividing by the total number of pregnancies, not including the proband.

Probability ( $\mathrm{p}$ ) values were obtained using the $\chi^{2}$ analysis for contingency tables with Yates's correction for continuity.

\section{Results and discussion}

In contrast to the BC study, recurrence rate in the Quebec sample was not significantly lower in the lower NTD group (table 1). It might be argued that the difference between the two centres resulted from differences in referral pattern. This would require that probands with affected sibs were less likely to be referred if they had lower rather than upper NTDs in BC but not in Quebec. This seems very unlikely. Another possibility would be that the recurrence rate in the Quebec lower NTD sample was falsely raised by the inclusion of upper cases misclassified as lower, but the difference from the BC value is too great for this explanation to be plausible. Alternatively, it may be that there is a real difference between high and low NTD frequency areas, with the high area (Quebec, 3.3 per 1000 livebirths) ${ }^{11}$ having a higher proportion of aetiologically different cases than the low area (BC, 1.6 per 1000 livebirths), ${ }^{12}$ but it is difficult to construct a model with a plausible admixture of cases with the appropriate properties. A fourth explanation may be that one or the other sample is manifesting a large deviation from the real mean through sampling error.

Our study also differed from that of Hall et $a l^{\beta}$ and that of Toriello and Higgins ${ }^{1}$ with respect to concordance as to type of defect among affected sibs. When an NTD recurred within a sibship, the recurrent case was concordant with respect to NTD type in only $50 \%$ of the sib pairs (table 2). This finding differed from the full concordance seen in Michigan, BC, and perhaps Hungary ${ }^{13}$ (where the one discordant pair could have been the result of chance), but was similar to that in studies from Newfoundland, ${ }^{14}$ Scotland, ${ }^{15}$ and England ${ }^{16}$ where an appreciable degree of discordance was also present.

A potential criticism of hospital based stud-

Table 2 Concordance of NTD type within sibships.

\begin{tabular}{lcc}
\hline Study population & No & Concordance (\%) \\
\hline Quebec* $^{*}$ & 18 & 50 \\
Newfoundland $^{14}$ & 11 & 64 \\
Scotland $^{15}$ & 48 & 69 \\
England $^{16}$ & 38 & 82 \\
Hungary $^{13}$ & 17 & 94 \\
Michigan $^{1}$ & 9 & 100 \\
BC $^{3}$ & 16 & 100
\end{tabular}

* The nine discordant sibships consisted of lumbar/lumbosacral SB in one sib and AN (six cases), EN (one), and cervical/ cranial meningomyelocele (two) in the other.
Table 3 Frequency of additional malformations in cases of NTD.

\begin{tabular}{|c|c|c|c|c|c|}
\hline \multirow[b]{2}{*}{ Study } & \multicolumn{2}{|c|}{ Upper NTD } & \multicolumn{2}{|c|}{ Lower NTD } & \multirow[b]{2}{*}{ p } \\
\hline & No & $\%$ & No & $\%$ & \\
\hline $\begin{array}{l}\text { BC }^{3} \\
\text { Michigan }^{1 *} \\
\text { Europe }^{9} \\
\text { Quebec }\end{array}$ & $\begin{array}{r}287 \\
40 \\
1459 \\
154\end{array}$ & $\begin{array}{l}20 \cdot 2 \\
22 \cdot 5 \\
17 \cdot 1 \\
13 \cdot 0\end{array}$ & $\begin{array}{l}225 \\
164 \\
609 \\
122\end{array}$ & $\begin{array}{r}6.7 \\
3.6 \\
10.5 \\
6.6\end{array}$ & $\begin{array}{c}<0.0001 \\
<0.0001 \\
<0.001 \\
\quad \text { NS }\end{array}$ \\
\hline
\end{tabular}

* AN cases were not included.

ies is the possible incomplete ascertainment of stillborn and aborted cases. This would result in a deficit of upper NTD cases (especially anencephalics). Whether or not it is this deficit that caused an inflation of sib concordance in previous studies, ${ }^{1313}$ it has little or no effect in our study.

Rates of additional malformation in upper and lower NTD probands were measured as well as spontaneous abortion rates in their mothers. Additional malformation rates were consistently more frequent in the upper than in the lower group, though not significantly so for Quebec (table 3). The BC sample showed no difference in abortion rate between upper and lower groups (table 4), both values being somewhat higher than that generally reported for the population $(15 \%) .{ }^{17}$ The high rate of abortion in the Michigan upper group deviates so much from other reports that it may well be an artefact of small sample size. However, the Quebec sample also shows a higher value for the upper than the lower group, possibly a further reflection of heterogeneity between high and low incidence areas.

The data in table 5 show an excess of females in the upper group and not in the lower group, the only exception being $\mathrm{BC}$. This may relate to the fact that $\mathrm{BC}$ is a low incidence area, since it has been shown previously that the sex ratio for NTDs decreases as NTD incidence increases. ${ }^{18}$

The general trend for a low sex ratio in upper but not lower NTDs could be explained by a hypothesis of Seller and Perkins-Cole. ${ }^{19}$ They found that female mouse embryos are developmentally retarded compared with males at the onset of neurulation. If this situation also exists in humans, it would make females more susceptible than males to the earlier arising upper NTDs if the 'critical period' for teratogenic insult was longer, or if neural tube closure was delayed more than that of the embryo in general.

To account for the high sex ratio observed in lower NTDs, it has been postulated that, during lower NTD development, a slight growth retardation in females could protect them from lower defects. ${ }^{4}$ Since canalisation (secondary neurulation) depends on the formation and

Table 4 Spontaneous abortion rate in mothers of NTD probands.

\begin{tabular}{lcccccc}
\hline & \multicolumn{2}{c}{ Upper NTD } & & \multicolumn{2}{c}{ Lower NTD } & \\
\cline { 2 - 3 } Study & No & $\%$ & & No & $\%$ & p \\
\hline BC $^{3}$ & 528 & $19 \cdot 7$ & & 338 & $18 \cdot 0$ & NS \\
Michigan $^{1 *}$ & 86 & $44 \cdot 2$ & & 262 & $18 \cdot 7$ & $<0.0001$ \\
Quebec $^{n n n n n n n}$ & 478 & $19 \cdot 2$ & & 403 & $9 \cdot 9$ & $<0.001$ \\
\hline
\end{tabular}

* AN cases were not included. 
Table 5 Sex ratios (SR) in probands with upper and lower NTDs.

\begin{tabular}{lccccc}
\hline & \multicolumn{2}{c}{ Upper NTD } & & \multicolumn{2}{c}{ Lower NTD } \\
\cline { 2 - 3 } \cline { 6 - 6 } & SR & No & & SR & No \\
\hline Quebec & 0.81 & 141 & & 1.07 & 122 \\
BC $^{3}$ & 0.81 & 271 & & 0.76 & 220 \\
Scotland $^{15}$ & $<1$ & 248 & & 1.32 & 51 \\
England $_{\text {Spain }}^{21}$ & 0.59 & 127 & & 3.00 & 20 \\
\hline
\end{tabular}

coalescence of vacuoles within masses of cells, the faster developing males might overproduce these vacuoles, making them more susceptible to lower defects.

However, in light of the recent conclusion that the cut off point between primary and secondary neurulation is probably as low as the second sacral segment, ${ }^{20}$ most NTDs are likely to be caused by abnormal primary neurulation (over $95 \%$ in our sample). If so, there is no need for an upper/lower distinction on the basis of different closure mechanisms. Nevertheless, epidemiological differences between defects of the upper and lower neural tube have been observed in this and previous studies. To explain these differences, we propose that (1) upper and lower NTDs share a similar pathogenesis but at different developmental stages, and (2) the embryo is more susceptible during early neurulation.

On this basis, the observed differences can still be explained without involving differences in closure mechanism. According to the multifactorial threshold hypothesis, ${ }^{10}$ females, being more retarded than males during early neurulation when the upper neural tube is closing, are more susceptible to adverse factors and therefore females are more prone to upper NTDs. This effect would have diminished or disappeared by the stage of lower neural tube closure. Also, embryos of either sex that have defective early neurulation may be more liable to have other developmental abnormalities resulting from disturbances of induction or neural crest migration, or other insults to the early embryo.

Clearly more studies are indicated to assess whether the above differences between upper and lower NTDs are real and how they relate to NTD incidence.

This work was supported by a Medical Genetics Group Grant from the Medical Research Council of Canada.

1 Toriello HV, Higgins JV. Possible causal heterogeneity in spina bifida cystica. Am f Med Genet 1985;21:13-20.

2 Copp AJ, Brook FA, Estibeiro P, Shum ASW, Cockcroft DL. The embryonic development of mammalian neural tube defects. Prog Neurobiol 1990;35:363-403.

3 Hall JG, Friedman JM, Kenna BA, Popkin J, Jawanda M, Arnold W. Clinical, genetic, and epidemiological factor in neural tube defects. Am f Hum Genet 1988;43:827-37.

4 Seller MJ. Neural tube defects and sex ratios. $\mathrm{Am} F \mathrm{Med}$ Genet 1987;26:699-707.

5 Lemire RJ, Loeser JD, Leech RW, Alvord EC. Normal and abnormal development of the human nervous system. New York: Harper and Row, 1975:54-83.

6 McCormick WF. Is anencephaly a single entity? A brief note on the morphologic heterogeneity of anencephaly. In: Bergsma D, ed. The nervous system. New York: Alan $\mathrm{R}$ Liss for the National Foundation - March of Dimes. Birth Defects 1971;VII(1):94-6.

7 Khoury MJ, Erickson JD, James LM. Etiologic heterogeneity of neural tube defects: clues from epidemiology. Am 7 Epidemiol 1982;115:538-48.

Am F Epidemiol 1982;115:538-48.
8 Seller MJ, Kalousek DK. Neural tube defects: heterogeneity and homogeneity. Am $\mathcal{F}$ Med Genet 1986;suppl

9 Dolk H, De Wals P, Gillerot Y, et al. Heterogeneity of neural tube defects in Europe: the significance of site of defect and presence of other major anomalies in relation to geographic differences in prevalence. Teratology 1991;44:547-59.

10 Nora JJ, Fraser FC. Medical genetics: principles and practice. 3rd ed. Philadelphia: Lea \& Febiger, 1989.

11 Horowitz I, McDonald AD. Anencephaly and spina bifida in the province of Quebec. Can Med Assoc 1969;100:748-55.

$12 \mathrm{McBride}$ ML. Sib risks of anencephaly and spina bifida in British Columbia. Am $\mathcal{f}$ Med Genet 1979;3:377-87.

13 Török O, Papp Z. Are the neurulation and canalization forms of neural tube defects causally distinct? $A m \mathcal{F} M e d$ Genet 1991;39:241.

14 Frecker MF, Fraser FC, Heneghan WD. Are 'upper' and 'lower' neural tube defects aetiologically different? $\mathcal{Y} \mathrm{Med}$ 'lower' neural tube defect

15 Drainer E, May HM, Tolmie JL. Do familial neural tube defects breed true? F Med Genet 1991;28:605-8.

16 Seller MJ. Neural tube defects: are neurulation and canalization forms causally distinct? $\mathrm{Am} \mathcal{f}$ Med Gene 1990;35:394-6

17 Warburton D, Fraser FC. Spontaneous abortion risks in man: data from reproductive histories collected in medical genetics unit. Am f Hum Genet 1964;16:1-25.

18 Frecker M, Fraser FC Epidemiological studies of neural tube defects in Newfoundland. Teratology 1987;36:355 61.

19 Seller MJ, Perkins-Cole KJ. Sex difference in mouse embryonic development at neurulation. $\boldsymbol{F}$ Reprod Fertil embryonic develop

20 Müller F, O'Rahilly $R$. The development of the human brain, the closure of the caudal neuropore, and the beginning of secondary neurulation at stage 12. Anat Embryol 1987;176:413-30

21 Martinez Frias ML, Parralo JA, Salvador J, Frias JL. Sex ratios in neural tube defects. Lancet 1986;ii:871-2. 